\title{
Image analysis and statistical evaluation of two-dimensional human eye retina images of healthy and glaucomatous eyes
}

\author{
František Pluhácé, Jaroslav Pospíśll
}

Department of Experimental Physics and Joint Laboratory of Optics of Palacký University and Institute of Physics of Academy of Sciences, 17. listopadu 50a, 77207 Olomouc, Czech Republic.

\begin{abstract}
In this paper, a new automatic glaucoma diagnostic method for determining the probability of glaucoma occurring in a eye under study is described. This method is based on computer analysis of two-dimensional images of the blind spot of the human eye retina and on successive statistical evaluation of the data obtained. First, the characteristic symptoms of glaucoma are shortly described. Next, a suitable numerical parameter of the retina blind spot is defined. The method of computer image analysis for automatic determination of the parameter in question is described and it is applied to a set of normal healthy eye images and to a set of glaucomatous eye images. The probability of glaucoma occurring for each value of the parameter introduced is suitably defined and computed by virtue of statistical evaluation of the results obtained.
\end{abstract}

Keywords: glaucoma, diagnostic method, pallor, image analysis, statistical evaluation.

\section{Introduction}

The monitoring of changes on the human eye retina enables recognising a number of grave diseases in their early stadium, for example, glaucoma, diabetes, hypertension and arteriosclerosis, [1]-[7]. At present, it is possible to scan the human eye retina using the fundus camera connected to a computer by a convenient graphic card. A digital image obtained this way can be advantageously archived on an appropriate data medium with the possibility of futher processing. This procedure is convenient especially in the case of glaucoma diagnostics.

Glaucoma is a grave eye disease characterised by an increased pressure within the eyeball and typical changes on the eye retina and in the eye visual field [1]-[5]. Some of the first symptoms of glaucoma are the typical changes on the blind spot of the retina (named papilla or optic disc-OD). Approximately in the centre of the optic disc there is a depression or a cup of optic disc, whereby the retina nutrient vessels pass. The size of the cup grows with glaucoma progression and is often used as an indicator of glaucoma. The size of the cup can be measured and evaluated by the method of computer evaluation of stereoimages or the method of laser scanning tomography, [8]-[15]. The bottom of the glaucomatous cup contains a bright area 

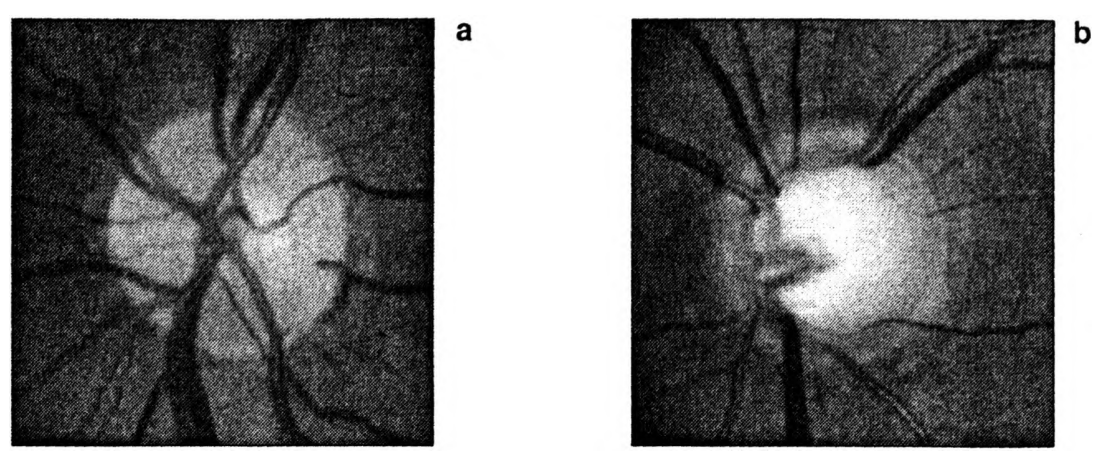

Fig. 1. Optic disc of a normal healthy eye (a) and the glaucomatous optic disc with the large cup and pallor (b).

named pallor, which represents a part of the cup with the total loss of the retina photosensitive cell nerve fibres and which grows with glaucoma progression. Thus, the pallor area can be used as a diagnostic indicator of glaucoma. The optic disc of a normal healthy eye and the glaucomatous optic disc are shown in Fig. 1. Present methods of pallor analysis [5], [8], [10], [15] mostly enable us to observe changes of the pallor shape and area with time. A new method of finding and statistical analysis of a representative pallor area on a two-dimensional colour computer digital image of OD is described in the following. In this method, the representative OD area and the representative pallor area on the image are determined using a suitable computer image analysis process and the data obtained are statistically evaluated for healthy and glaucomatous eye images. The ratio of the representative pallor area and the representative $\mathrm{OD}$ area (denoted as $\mathrm{P} / \mathrm{D}$ area ratio or only $\mathrm{P} / \mathrm{D}$ ) is used as the diagnostic indicator of glaucoma. All numerical calculations were performed using mathematical software MATLAB and MATHCAD.

\section{Determination of the optic disc representative area and pallor representative area in the image}

The present method designed for determination of the optic disc representative area and the pallor representative area in the image uses the two-dimensional colour computer digital image obtained by a currently used colour fundus camera connected to a computer. The representative margin of OD is approximated by a boundary ellipse, fitted by six points in the colour digitised image of OD, selected by the operator. The area within the boundary ellipse is the representative OD area. The pole $O$ of the polar coordinate system used is in the centre of the aforementioned representative boundary ellipse and the polar line is oriented horizontally in the reverse direction to the nose. The angle is measured clockwise in the case of the right eye and anticlockwise in the case of the left eye.

The colour digital computer image consists of three colour channels: red, green and blue. The values of brightness of each colour channel are usually quantized to 256 
levels and are represented by integer numbers from 0 to 255 . The value 0 represents the minimum brightness and the value 255 represents the maximum brightness of the given colour channel of the colour digital image. The brightness values represented by the value 255 are mostly considered as saturated brightness values. The saturation of the image brightness in the optic disc area makes it impossible to effectively use the pallor-detecting algorithm described in the following text. It has been ascertained experimentally that the brightness value of the green channel of the optic disc image is not saturated in the optic disc area and the pallor area is displayed in the highest contrast in this colour channel. Thus, only the green channel of the digital OD image under study is used to find the pallor area. The brightness of the OD image generally undesirably depends on the illumination of OD during taking the photograph. Hence, the brightness values $G$ of the green image channel are suitably normalised. First, two pixel groups $A_{1}$ and $A_{2}$ are defined. The image point (pixel) $(u, v)$ located at the intersection of the $u$-th row and the $v$-th column of the image lies within these pixel groups just when the inequality holds

$$
r(\phi)-\frac{a}{5} \leq \sqrt{\left(u-u_{\mathrm{O}}\right)^{2}+\left(v-v_{\mathrm{O}}\right)^{2}} \leq r(\phi)
$$

where: $-15^{\circ} \leq \phi \leq 15^{\circ}$ in the case of group $A_{1}$ or $165^{\circ} \leq \phi \leq 195^{\circ}$ in the case of group $A_{2}$ (see Fig. 2). The point $\left(u_{\mathrm{O}}, v_{\mathrm{O}}\right)$ represents the position of the pole $\mathrm{O}$ of the above defined polar coordinate system, $a$ is the semimajor axis size of the boundary ellipse, $\phi$ - the oriented angle included with the position vector of the pixel $(u, v)$ and the polar line, $r$ - the mutual distance between the pole $\mathrm{O}$ and a boundary ellipse point with the angular coordinate $\phi$ in the defined coordinate system. The brightness values of the $\mathrm{OD}$ image areas corresponding to pixel groups $A_{1}, A_{2}$ are not practically influenced by the gradual glaucoma progression and can be used to determine the reference brightness values. Two reference brightness values $G_{1}$ and $G_{2}$ are defined as the green

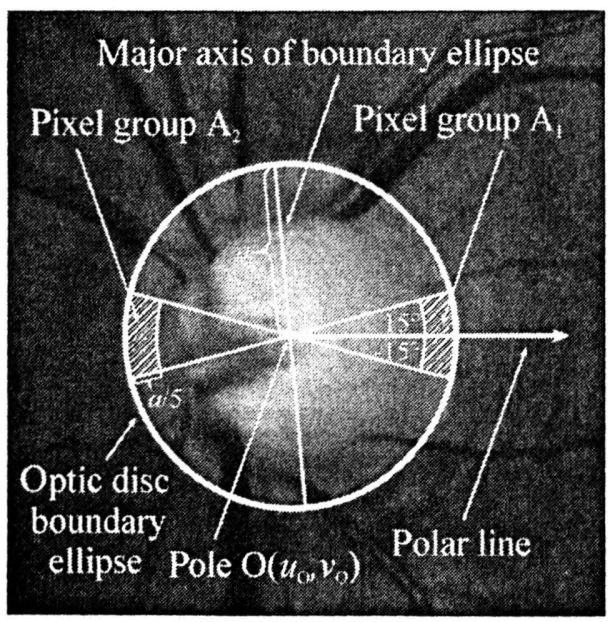

Fig. 2. Optic disc boundary ellipse, the polar coordinate system and the pixel groups $A_{1}$ and $A_{2}$. 
image channel brightness values with the highest occurrence frequency in the pixels of groups $A_{1}$, and $A_{2}$, respectively. The location of each pixel group is represented by either the average column and row indices $\bar{u}_{1}$ and $\bar{v}_{1}$, or $\bar{u}_{2}$ and $\bar{v}_{2}$ of the group pixels. The required normalised value $G_{\mathrm{n}}(u, v)$ of the brightness at each point $(u, v)$ of the OD image is defined by the relation

$$
G_{\mathrm{n}}(u, v)=\frac{2 G(u, v)}{G_{1}+G_{2}}
$$

The values $G_{1}$ and $G_{2}$ differ in general, since the brightness of the OD periphery inconveniently increases in the polar line direction. This effect can be effectively compensated by an acceptable brightness transformation

$$
G_{\mathrm{n}}^{\prime}(u, v)=G_{\mathrm{n}}(u, v)-G_{1 \mathrm{n}}-\frac{G_{2 \mathrm{n}}-G_{1 \mathrm{n}}}{\bar{v}_{2}-\bar{v}_{1}}\left(v-\bar{v}_{1}\right)
$$

of normalised brightness $G_{\mathrm{n}}(u, v)$ in the pixel $(u, v)$ to the new value $G_{\mathrm{n}}^{\prime}(u, v)$. The quantities $G_{1 \mathrm{n}}$ and $G_{2 \mathrm{n}}$ are the values of $G_{1}$ and $G_{2}$ normalised by Eq. (2). The representative pallor area in the image is defined as the image area within the boundary ellipse, where the normalised transformed value $G_{\mathrm{n}}^{\prime}$ is greater than or equal to the suitable selected threshold value $p$. The equality $p=0.2$ is considered in the following text.

\section{Experimental results and their statistical evaluation}

The colour digital computer images of OD of 240 eyes of 120 normal healthy subjects and 240 glaucomatous eyes of 120 subjects with glaucoma were used in this study. The computer digital images of OD were obtained by the computer-connected digital fundus camera DFK 98. The digital fundus camera consists of the colour CCD video camera connected to the adapted Retinofot Carl Zeiss Jena 201. The size of the photographed optic disc area was approximately $5.8 \times 4.4 \mathrm{~mm}$ and the size of the digital image obtained was $768 \times 576$ pixels. Brightness values of each colour channel were quantized to 256 discrete levels. Futher considerations and results relate only to the above-mentioned image parameters. The diagnoses were determined by the glaucoma -specialised ophthalmologist by virtue of the measurement of the intraocular tension, eye visual field, enlargement of the cup using the laser scanning tomograph and a subjective investigation of the retina nerve fibre layer thickness. The age of the normal healthy subjects ranged from 14 to 76 years with the mean age of 46 years and with the standard deviation of the age of 13 years. The age of the glaucomatous subjects ranged from 10 to 82 years with the mean age of 51 years and with the standard deviation of the age of 16 years.

For all the images introduced, the required $P / D$ area ratio was calculated for the entire representative OD area. The mean values of the P/D area ratio were 0.10 in the case of healthy eyes and 0.24 in the case of glaucomatous eyes and standard deviations 
were 0.07 and 0.09 . Thus, according to the presumption it is shown that the P/D area ratio is really markedly influenced by glaucoma and can be used to the diagnosis determination.

\subsection{Dependence of $P / D$ area ratio on age and manual optic disc demarcation}

The dependences of the P/D area ratio on age (in years) of the photographed healthy subjects and glaucomatous subjects were approximated by the lines using the regression analysis [16], [17]. For healthy subjects, the slope was $-5.6 \times 10^{-5}$, the intercept was 0.1 and the correlation coefficient was -0.01 . For glaucomatous subjects, the slope was $-1.7 \times 10^{-3}$, the intercept was 0.3 and the correlation coefficient was -0.3 . The small values of slopes indicate that the age dependences of P/D area ratio are insignificant.

The manual demarcation of the optic disc border was repeated five-times for 10 randomly selected healthy OD images and 10 randomly selected glaucomatous OD images. For each demarcation of the optic disc border, the required P/D area ratio was computed. Next, for each selected image, the ratio of the P/D standard deviation of $\mathrm{P} / \mathrm{D}$ to mean value of P/D were computed. The variability $\delta$ of the P/D area ratio measurement was defined as the mean value of all introduced relative standard deviations $\delta=0.07$.

\subsection{Definition of glaucoma risk-classes}

The probability densities $p_{\mathrm{h}}$ and $p_{\mathrm{g}}$ of the experimentally obtained statistical distributions of the P/D area ratio values of the above-mentioned healthy and glaucomatous eye images can be approximated by the experimentally ascertained relations:

$$
\begin{array}{ll}
p_{\mathrm{h}}(\mathrm{P} / \mathrm{D})=N_{\mathrm{h}} \exp \left[-\beta_{\mathrm{h} 1}\left(\mathrm{P} / \mathrm{D}-\beta_{\mathrm{h} 2}\right)^{3}\right], & \mathrm{P} / \mathrm{D} \in\langle 0,1\rangle, \\
p_{\mathrm{g}}(\mathrm{P} / \mathrm{D})=N_{\mathrm{g}} \exp \left[-\beta_{\mathrm{g} 1}\left(\mathrm{P} / \mathrm{D}-\beta_{\mathrm{g} 2}\right)^{2}\right], & \mathrm{P} / \mathrm{D} \in\langle 0,1\rangle
\end{array}
$$

where:

$$
\begin{aligned}
& N_{\mathrm{h}}=1 / \int_{0}^{1} \exp \left[-\beta_{\mathrm{h} 1}\left(x-\beta_{\mathrm{h} 2}\right)^{3}\right] \mathrm{d} x, \\
& N_{\mathrm{g}}=1 / \int_{0}^{1} \exp \left[-\beta_{\mathrm{g} 1}\left(x-\beta_{\mathrm{g} 2}\right)^{2}\right] \mathrm{d} x
\end{aligned}
$$

are the normalisation constants, and $\beta_{\mathrm{h} 1}, \beta_{\mathrm{h} 2}, \beta_{\mathrm{g} 1}, \beta_{\mathrm{g} 2}$ (shortly $\beta_{\mathrm{h} 1,2}$ and $\beta_{\mathrm{g} 1,2}$ ) are real parameters. The functions (4) and (5) were fitted by histograms of $P / D$ area ratio values of healthy eye images and glaucomatous eye images using the least squares method [16]. For each histogram, 10 class intervals were used. All computations were performed numerically with a sufficient accuracy. The obtained estimations of 
T a bl e. Estimated values of parameters $\beta_{\mathrm{hl}, 2}, \beta_{\mathrm{gl}, 2}$ and their standard deviations $\sigma_{\mathrm{hl}, 2}, \sigma_{\mathrm{gl}, 2}$ obtained using the least squares method.

\begin{tabular}{llll}
\hline Parameter & Estimated value & Standard deviation & Estimated value \\
\hline$\beta_{\mathrm{h} 1}$ & $7.0 \times 10^{2}$ & $\sigma_{\mathrm{h} 1}$ & $0.6 \times 10^{2}$ \\
$\beta_{\mathrm{h} 2}$ & $11.4 \times 10^{-2}$ & $\sigma_{\mathrm{h} 2}$ & $0.4 \times 10^{-2}$ \\
$\beta_{\mathrm{g} 1}$ & $5.2 \times 10$ & $\sigma_{\mathrm{g} 1}$ & $0.4 \times 10$ \\
$\beta_{\mathrm{g} 2}$ & $24.3 \times 10^{-2}$ & $\sigma_{\mathrm{g} 2}$ & $0.4 \times 10^{-2}$ \\
\hline
\end{tabular}

parameters $\beta_{\mathrm{h} 1,2}, \beta_{\mathrm{g} 1,2}$ and their standard deviations $\sigma_{\mathrm{h} 1}, \sigma_{\mathrm{h} 2}, \sigma_{\mathrm{g} 1}, \sigma_{\mathrm{g} 2}$ (shortly $\sigma_{\mathrm{h} 1,2}$ and $\left.\sigma_{\mathrm{g} 1,2}\right)$ are introduced in the Table. Both fitted probability densities were tested by chi-squared test of fit [16], [17] and they did not reject for significance levels smaller than $22 \%$ in the case of $p_{\mathrm{h}}$ and smaller than $42 \%$ in the case of $p_{\mathrm{g}}$. Histograms of healthy and glaucomatous eye images $\mathrm{P} / \mathrm{D}$ area ratio with fitted probability densities $p_{\mathrm{h}}, p_{\mathrm{g}}$ are presented in Figs. 3, 4.

Next, three glaucoma risk classes of eyes were defined: low-glaucoma-risk class, glaucoma-suspected class and high-glaucoma-risk class. The low-glaucoma-risk class includes eyes with the P/D area ratio under $t_{l}$, the glaucoma-suspected class includes eyes with the $\mathrm{P} / \mathrm{D}$ area ratio from interval $\left\langle t_{1}, t_{2}\right\rangle$ and the high-glaucoma-risk class includes eyes with the P/D area ratio over $t_{2}$, where the parameters $t_{1}$ and $t_{2}$, $0<t_{1}<t_{2}<1$, are suitably chosen values of P/D area ratio, see Fig. 5. Under these conditions, the glaucomatous eye is inconveniently classified to the low-glaucoma-risk class with the probability

$$
P_{\mathrm{g} / \text { low }}=\int_{0}^{t_{1}} p_{\mathrm{g}}(x) \mathrm{d} x,
$$

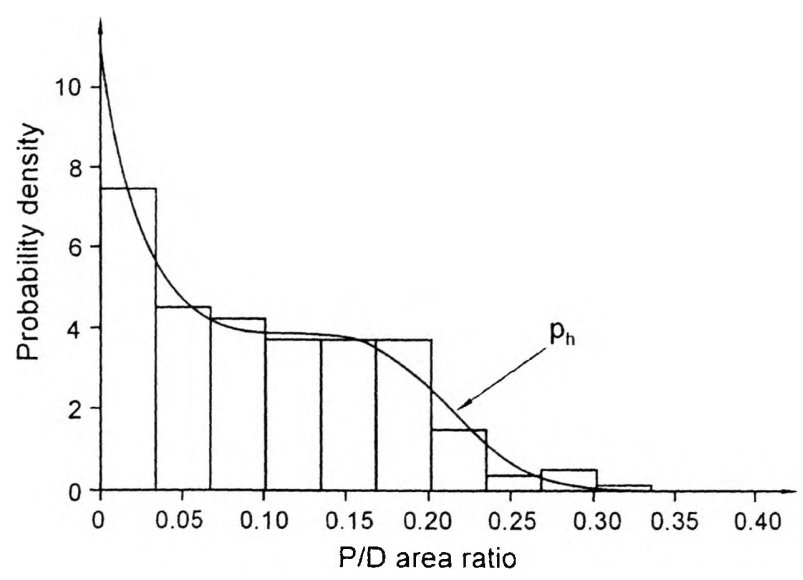

Fig. 3. Histogram of the healthy eye images $\mathrm{P} / \mathrm{D}$ area ratio with the fitted probability density $p_{\mathrm{h}}$. 


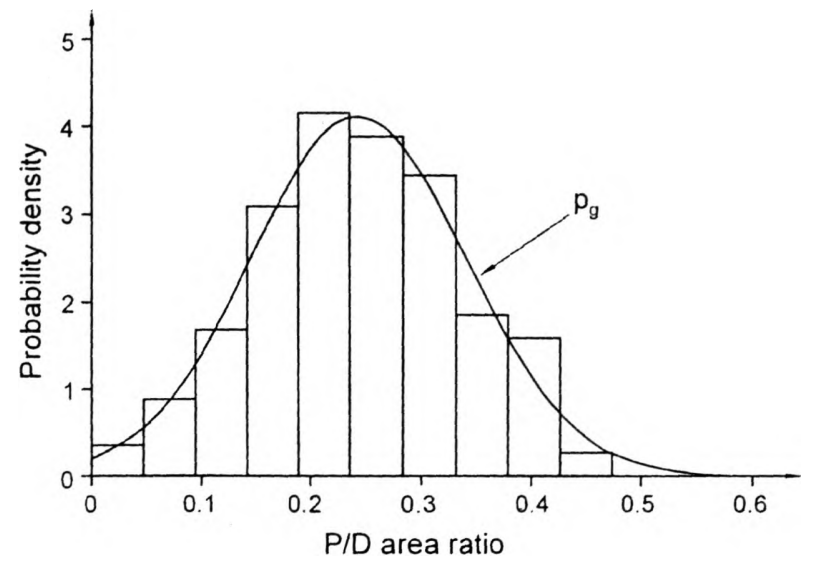

Fig. 4. Histogram of the glaucomatous eye images $P / D$ area ratio with the fitted probability density $p_{g}$.

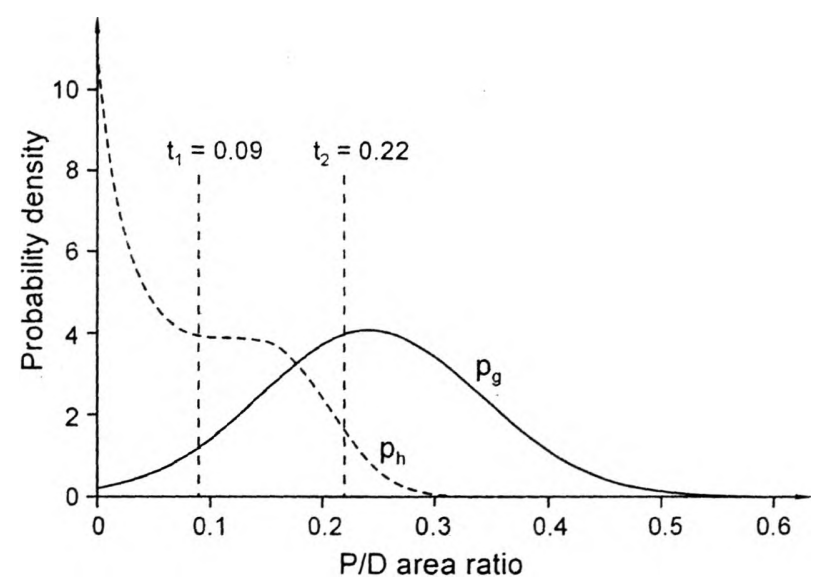

Fig. 5. Graphical dependences of $p_{\mathrm{l}}, p_{\mathrm{g}}$ on P/D area ratio for values of $\beta_{\mathrm{h} 1,2}$ and $\beta_{\mathrm{g} 1,2}$ introduced in the Table. The chosen values $t_{1}=0.09$ and $t_{2}=0.22$ of parameters $t_{1}$ and $t_{2}$ are marked.

and the healthy eye is inconveniently classified to the high-glaucoma-risk class with the probability

$$
P_{\mathrm{h} / \mathrm{high}}=\int_{t_{2}}^{1} p_{\mathrm{h}}(x) \mathrm{d} x .
$$

The values of $P_{\mathrm{g} / \mathrm{low}}$, respectively $P_{\mathrm{h} / \mathrm{high}}$, must be small enough for practical utilization of the above-mentioned eye classification. In the case of small values of $P_{\mathrm{g} / \mathrm{low}}$ and $P_{\mathrm{h} / \mathrm{high}}$, eyes from the low-glaucoma-risk class can be diagnosed as healthy with the probability of the diagnosis being mistakenly determined equal to $P_{\mathrm{g} / \text { low }}$, respectively eyes from the high-glaucoma-risk class can be diagnosed as glaucomatous 
with the probability of mistaken diagnosis equal to $P_{\mathrm{h} / \mathrm{high}}$. With respect to the variability of $\mathrm{P} / \mathrm{D}$ area ratio measurement and non-zero values of $\sigma_{\mathrm{h} 1,2}$ and $\sigma_{\mathrm{g} 1,2}$, the probabilities $P_{\mathrm{l} / \text { high }}, P_{\mathrm{g} / \text { low }}$ are determined with the relevant standard deviations $\sigma_{\mathrm{g} / \mathrm{low}}$, $\sigma_{\mathrm{h} / \mathrm{high}}$. The numerical analysis shows that the influence of $\sigma_{\mathrm{h} 1,2}$ and $\sigma_{\mathrm{g} 1.2}$ on $\sigma_{\mathrm{g} / \text { low }}$, $\sigma_{\mathrm{h} / \mathrm{high}}$ is negligible and need not be considered. According to the common error -propagation rule, it holds approximately

$$
\begin{aligned}
& \sigma_{\mathrm{h} / \mathrm{high}} \approx\left|\frac{\partial P_{\mathrm{h} / \mathrm{high}}}{\partial t_{2}} \delta t_{2}\right|, \\
& \sigma_{\mathrm{g} / \text { low }} \approx\left|\frac{\partial P_{\mathrm{g} / \text { low }}}{\partial t_{1}} \delta t_{1}\right| .
\end{aligned}
$$

For the chosen values $t_{1}=0.09, t_{2}=0.22$, the equalities $P_{\mathrm{h} / \mathrm{high}}=0.05, \sigma_{\mathrm{h} / \mathrm{high}} \approx 0.03$, $P_{\text {g/low }}=0.05, \sigma_{\text {g/low }} \approx 0.01$ are valid.

The suspected probability $P_{\mathrm{h} / \text { susp }}$ of classifying a healthy eye to the glaucoma -suspected class and the probability $P_{\mathrm{g} / \mathrm{susp}}$ of a glaucomatous eye being classified to the glaucoma-suspected class are fulfilled by the relations:

$$
\begin{aligned}
P_{\mathrm{h} / \text { susp }} & =\int_{t_{1}}^{t_{2}} p_{\mathrm{h}}(x) \mathrm{d} x, \\
P_{\text {g/susp }} & =\int_{t_{1}}^{t_{2}} p_{\mathrm{g}}(x) \mathrm{d} x .
\end{aligned}
$$

The standard deviations $\sigma_{\mathrm{g} / \mathrm{susp}}$ and $\sigma_{\mathrm{h} / \mathrm{susp}}$ of $P_{\mathrm{h} / \mathrm{susp}}$ and $P_{\mathrm{g} / \mathrm{susp}}$ can be computed again using the aforementioned error-propagation rule:

$$
\begin{gathered}
\sigma_{\mathrm{h} / \text { susp }} \approx \sqrt{\left(\frac{\partial P_{\mathrm{h} / \text { susp }}}{\partial t_{1}} \delta t_{1}\right)^{2}+\left(\frac{\partial P_{\mathrm{h} / \mathrm{susp}}}{\partial t_{2}} \delta t_{2}\right)^{2}}, \\
\sigma_{\mathrm{g} / \text { susp }} \approx \sqrt{\left(\frac{\partial P_{\mathrm{g} / \text { susp }}}{\partial t_{1}} \delta t_{1}\right)^{2}+\left(\frac{\partial P_{\mathrm{g} / \mathrm{susp}}}{\partial t_{2}} \delta t_{2}\right)^{2}} .
\end{gathered}
$$

In the case of equalities $t_{1}=0.09, t_{2}=0.22$ being considered, the values $P_{\mathrm{h} / \mathrm{susp}}=0.45$, $\sigma_{\mathrm{h} / \mathrm{susp}} \approx 0.04, P_{\mathrm{g} / \mathrm{susp}}=0.34$ and $\sigma_{\mathrm{h} / \mathrm{susp}} \approx 0.06$ were obtained. It is evident that for eyes from the glaucoma-suspected class, the diagnosis cannot be determined using only the method described. It is necessary to take advantage of other investigations. 


\subsection{Determination of glaucoma occurrence probability on $P / D$ area ratio}

For the probability $P$ of the occurrence of glaucomatous eyes in the population of eyes with the given $P / D$ area ratio value (shortly glaucoma occurrence probability), the formula

$$
P=\frac{P_{\mathrm{p}} p_{\mathrm{g}}}{P_{\mathrm{p}} p_{\mathrm{g}}+\left(1-P_{\mathrm{p}}\right) p_{\mathrm{h}}},
$$

can be derived on the basis of the relations of the conditional probability theory [17]. The probability densities $p_{\mathrm{h}}$ and $p_{\mathrm{g}}$ are given by Eqs. (4) and (5). The parameter $P_{\mathrm{p}}$ with the standard deviation $\sigma_{P_{\mathrm{p}}}$ means the probability of glaucoma occurring in the whole population (without reference to the $P / D$ area ratio value) of a given age range. In general, parameter $P_{\mathrm{p}}$ is markedly influenced by the race of the investigated subjects and differs for different age groups, see [1], [18]. The standard deviation $\sigma_{P}$ was estimated according to the error-propagation rule mentioned previously as follows:

$$
\sigma_{P} \approx \sqrt{\left(\frac{\partial P}{\partial(\mathrm{P} / \mathrm{D})}\right)^{2}(\delta \mathrm{P} / \mathrm{D})^{2}+\left(\frac{\partial P}{\partial P_{\mathrm{p}}}\right)^{2} \sigma_{P_{\mathrm{p}}}^{2}} .
$$

The influence of $\sigma_{\mathrm{h} 1,2}$ and $\sigma_{\mathrm{g} 1,2}$ was neglected. Formulas (16) and (17) are plotted as the functions of $P / D$ area ratio in Fig. 6 for two different age groups of white population (under 65 years and over 75 years) and for estimated values of $\beta_{\mathrm{h} 1,2}, \beta_{\mathrm{gl}, 2}$, $\sigma_{\mathrm{h} 1,2}$ and $\sigma_{\mathrm{g} 1,2}$ introduced in the Table. In the case of the age group under 65 years, the equalities $P_{\mathrm{p}}=0.01, \sigma_{P_{\mathrm{p}}}=0.005$ are valid and for the age group over 75 years, the equalities $P_{\mathrm{p}}=0.03, \sigma_{P_{\mathrm{p}}}=0.01$ are valid, see [18]. It is evident from the graph that

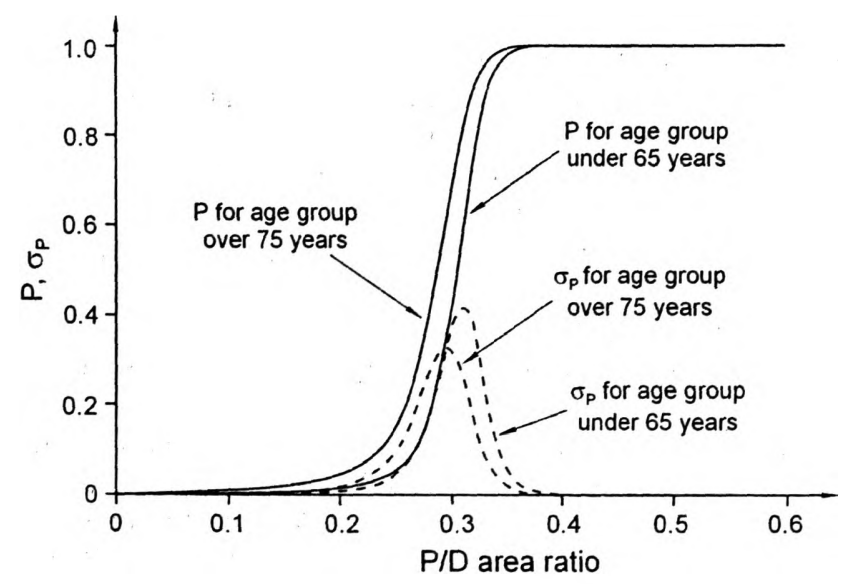

Fig. 6. Graphical dependences of $P$ and $\sigma_{P}$ on P/D area ratio for two different age groups of white population and for the values of $\beta_{\mathrm{hl}, 2}, \beta_{\mathrm{gl}, 2}, \sigma_{\mathrm{hl}, 2}$ and $\sigma_{\mathrm{gl}, 2}$ introduced in the Table. 
the glaucoma occurrence probability increases with an increased $\mathrm{P} / \mathrm{D}$ area ratio in both cases. The standard deviation $\sigma_{P}$ is inconveniently high, mainly in the interval of the maximal slope of $P$.

\section{Conclusions}

In this article, the statistical analysis performed validates the supposed influence of glaucoma on the parameter computed (P/D area ratio) using the designed image analysis algorithm, which represents the relative pallor area size. The statistical analysis of the parameter in question makes it possible to distinguish three glaucoma -risk classes. According to this classification, the diagnostic method was designed and possible diagnostic mistakes were analysed. By virtue of the experimentally obtained data, the dependence of the glaucoma occurrence probability on the P/D area ratio is found and presented in an analytical form. This dependence is numerically and graphically evaluated for two different age groups of white population.

The method of image analysis and statistical evaluation of two-dimensional colour digital images of the optic disc can be used as a useful tool for glaucoma diagnostics. This method is able to substitute a subjective evaluation of glaucoma damage of the optic disc.

Acknowledgment - The authors would like to take this opportunity to thank Dr. Tomáš Kuběna for providing all optic disc images used in this investigation.

\section{References}

[1] Shields B., Textbook of Glaucoma, Williams \& Wilkins, Baltimore 1992.

[2] Hanuš K., Boguszaková J., Diblik P., Dotrélová D., Filipec M., Hycl J. Karel I., Kocour I., Kraus H., KŘepelková J., Kuchynka P., Otradovec J., Palećková J., Peregrin J., Řehúréek J., Svèrák J., ŚEDivÝ A., Compendium of Eye Medicine, Grada, Praha 1997 (in Czech).

[3] Nesterov A. P., Primary Glaucoma, Avicenum, Praha 1991 (in Czech).

[4] Ř̈hák S., Knobloch R., Riebel O., Vrabec F., Medicine of the Eye, Avicenum, Praha 1980 (in Czech).

[5] Nagin P., Schwartz B., Ophthalmology 92 (1985), 252.

[6] Nover A., The Ocular Fundus, Methods of Examination and Typical Findings, Schattauer, Stuttgart 1987.

[7] Sauter H., Straub W., Rossmann H., Atlas of the Ocular Fundus, Photographs of Typical Changes in Ocular and Systemic Disease, Urban \& Schwarzenberg, München 1997.

[8] Cornsweet T. N., Hersh S., Humphries J.C., Beesmer R.J., Cornsweet D.W, Quantification of the shape and color of the optic nerve head, [In] Advances in Diagnostic Visual Optics, G.M. Breinin, I.M. Siegel, Springer-Verlag, New York 1983, pp. 141-149.

[9] Cornsweet T.N., Hersh S., Ocular-Fundus Analyze, U.S. Patent No. 4715703, U.S. Government, Washington 1987.

[10] Pluhácek F., Pospišll J., Fine Mechanics and Optics 46 (2001), 326 (in Czech). 
[11] SHIOIRI T., Ophthalmologic Image Processor, U.S. Patent No. 6104828, U.S. Government, Washington 2000.

[12] Mikelberg F. S., Wijsman K., Schulzer M., J. Glaucoma 2 (1993), 101.

[13] ZiNSER G., HARBARTH U., SCHRÖDER H., Formation and analysis of three-dimensional data with the laser tomographic scanner (LST), [In] Scanning Laser Ophthalmoscopy and Tomography, J.E. Nasemann, R.O.W. Burk, Quintessenz Velrlag, München 1990 pp. 243-252.

[14] Bartz-Schmidt K. U., Sengersdorf A., Esser P., Walter P., Hilgers R.D., Krieglstein G.K., Greafe's Archive for Clinical and Experimental Ophthalmology 234 (1996), 227.

[15] Varma R., Spaeth G.L., Ophthalmic Surgery 19 (1988), 183.

[16] Kubáćek L., Kubáćovó L., Statistics and Metrology, Palacký University Press, Olomouc 2000 (in Czech).

[17] ŠKrášek J., TICHÝ Z., Principles of Applied Mathematics III, SNTL, Praha 1990 (in Czech).

[18] Klein B.E.K., Klein R., Sponsel W.E., Franke T., Cantor L.B., Martone J., Ophthalmology 99 (1992), 1499.

Received September 19, 2002 in revised form December 11, 2002 